\title{
Identification for prognostic value of differentially expressed genes in immune microenvironment of clear cell renal cell carcinoma
}

\section{Xingming Zhang}

Sichuan University West China Hospital

Xiaoxue Yin

Sichuan University West China Hospital

Zhenhua Liu

Sichuan University West China Hospital

Guangxi Sun

Sichuan University West China Hospital

Xudong Zhu

Sichuan University West China Hospital

Haoran Zhang

Sichuan University West China Hospital

Sha Zhu

Sichuan University West China Hospital

Jinge Zhao

Sichuan University West China Hospital Junru Chen

Sichuan University West China Hospital

Ni Chen

Sichuan University West China Hospital

\section{Pengfei Shen}

Sichuan University West China Hospital

Jia Wang

Sichuan University West China Hospital

Hao Zeng ( $\square$ kucaizeng@163.com )

https://orcid.org/0000-0003-0818-8151

\section{Research article}

Keywords: renal cell carcinoma, clear cell, immune microenvironment, differentially expressed genes, prognosis 
Posted Date: October 24th, 2019

DOI: https://doi.org/10.21203/rs.2.16420/v1

License: (c) (i) This work is licensed under a Creative Commons Attribution 4.0 International License. Read Full License 


\section{Abstract}

Background: Genes related to Anchorimmune microenvironment of clear cell renal cell carcinoma (cCRCC) remains unclear. We aimed to identify related to immune microenvironment and to screen the most significant genes to predict outcomes of ccRCC.

Methods: Gene expression and clinicopathological data from TCGA data portal were obtained (KIRC). Immune and stromal scores were calculated based on ESTIMATE algorithm. DEGs between low and high groups of immune scores were identified. Subsequent functional enrichment analysis and protein-protein interaction of DEGs were conducted by DAVID database.

Results: Patients were divided into low and high groups by medians according to immune (median: 1038.45) and stromal scores (median: 667.945), respectively. Immune scores were significantly correlated with clinicopathological parameters and overall survival (OS). Based on immune scores, 1433 genes were up-regulated, and among them, $890 \mathrm{DEGs}$ were significantly associated with OS. Based on top 10 DEGs, cases with number of up-regulated genes $\geq 5$ were associated poor OS $(P=0.002)$. In addition, the mean differences of percentages of CD8 T cells (11.32\%), CD 4 memory resting $T$ cells $(-4.52 \%)$ and mast resting cells $(-3.55 \%)$ between low and high immune scores were the most significant.

Conclusions: A list of immune microenvironment-related genes in ccRCC was initially identified, and high immune score was an independent poor prognostic factor of OS. Furthermore, the combination of these genes might use to predict the efficacy of immunotherapy. Further analyses of these genes were warrant to explore their potential association with the prognosis of ccRCC.

\section{Introduction}

Renal cell carcinoma (RCC) is one of the most common malignancies in urological oncology, among which clear cell RCC (ccRCC) is the most predominant histological type, accounting for more than $80 \%$ of all RCCs(1). Generally, ccRCC has been proved to be with highly infiltrated immune cells(2). Meanwhile, a subset of spontaneous regression of RCC, with a frequency of approximate $1 \%$, were attributed to immune-mediated(3). Therefore, ccRCC was one of the earliest malignancies in history to be treated with immunotherapy, and heretofore remains one of most responsive tumors(4-6). In recent years, the progress of molecular immunology has promoted the discovery of immune checkpoint inhibitors (ICls), which were successfully applied into clinical practice. Although complete response dramatically occurred up to $9 \%$ in patients with immunotherapy(7), still only part of population could benefit from either immunotherapy alone or immunotherapy combined with TKIs(ORR: 25-59\%)(7-12). Commonly speaking, response to immunotherapy in the field of cCRCC were not satisfied as we expected(13). This has prompted researchers around the world to keep exploring new potential biomarkers which can predict efficacy of immunotherapy and help clinical decision making.

Currently no optimal biomarker has been demonstrated to be capable to screen appropriate patients for immunotherapy in ccRCC. PD-L1 was the first biomarker to be considered as a promising predictor on 
immunotherapy. It has been proven that PD-L1 was a valuable biomarker in predicting immunotherapy efficacy of melanoma and lung cancer(14). Unfortunately, PD-L1 expression failed to distinguish appropriate patients with ccRCC for ICls(14).Although a variety of prognostic models have been used in clinical settings to predict therapeutic effects and guide clinical decisions of RCC(15), these models offers limited information, especially in the era of targeted therapy and even immunotherapy. Theoretically, tumor mutation burden (TMB) and tumor neoantigen burden (TNB) could predict tumor response to immunotherapy. In terms of $\mathrm{cCRCC}$, only a modest mutation frequency (with a median of $2.6 / \mathrm{Mb}$, ranged from $0.1-10 / \mathrm{Mb}$ ) observed $(16,17)$. Recent study has demonstrated all three classic histopathologic types of RCC have predominantly high level of TNB(17), the key problem was it was hard to calculate accurately. In the study of IMmotion150, TNB failed to predict the immunotherapy efficacy of mRCC (18). In addition, TMB is positively correlated with cytolytic activity (CYT) in most tumors and the CYT of CCRCC was the highest among 17 tumor types, while the correlation between TMB and CYT in ccRCC was not significant(19). As a whole, the above evidence suggested that none of current biomarkers could determine immune infiltration and predict immunotherapy response(16).

In the context of immuno-oncology, growing evidences suggested that tumor microenvironment (TME) plays an important role in tumor progression, metastasis and therapeutic resistance(20,21). Immune and stromal cells are the two main types of non-tumor cells in $\operatorname{TME}(22)$. It has been reported that regulatory $T$ cells (Tregs), tumor associated macrophages (TAM) and CD8 + T cells have different effects on tumor cells(23-27). Meanwhile, multiple researches have demonstrated that immune microenvironment is closely related to $\operatorname{ccRCC}(28-30)$. Whereas, the mechanisms of TME highly infiltrated with immune cells, spontaneous tumor remission in the course of treatment of primary tumor and the treatment response to immunotherapy in ccRCC still remain obscure. Since the immune microenvironment is a complex space consisted of various kinds of immune cells, the identification of these cells alone is insufficient to assess the complex microenvironment, which is mainly characterized by histology (immunohistochemistry, flow cytometry, etc.). Considering the technique is limited by multiple factors, such as the difficulty to simultaneously assess the number of miscellaneous cell types and the high demanded amount of tissue, Yoshihara et al. designed a calculation to quantitatively predict immune and stromal scores based on ESTIMATE algorithm (Estimation of STromal and Immune cells in MAlignant Tumor tissues using Expression data), which utilizes specific genes in immune and stromal cells to predict scores of tumor purity and infiltration extent(31, 32).

The cancer genome atlas (TCGA) is an open database aims to apply high-throughput genomic sequencing techniques to assist better understanding of cancer and thus improve the ability to prevent, diagnose and treatment of cancer $(33,34)$. In order to analyze the influences of immune and stromal related genes on the prognosis of $\mathrm{CCRCC}$, we aimed to classify $\mathrm{CCRCC}$ cases of TCGA into low and high score groups based on immune and stromal scores. Subsequently, we planned to identify differentially expressed genes between low and high groups and to screen the most significant genes to predict outcomes of ccRCC. 


\section{Materials And Methods}

\section{Acquisition of gene expression profile data}

We obtained level 3 gene expression profile of cCRCC patients (KIRC) from the TCGA data portal (https://tcga-data.nci.nih.gov/tcga/). In addition, clinical and pathological data were also down loaded. The presence of infiltrating stromal/immune cells in tumor tissues of ccRCC was predicted by a tool of ESTIMATE algorithm created by Yoshihara et al.

\section{Relationship between immune/stromal score and clinicopathological parameters and overall survival (OS)}

Based on the immune/stromal score mentioned before, we classified cases of ccRCC into low and high groups by medians according to immune and stromal scores, respectively. Subsequently, we analyzed correlations between immune or stromal score and T stage, histologic type, tumor stage and top 4 mutated genes in cCRCC (VHL, PBRM1, SETD2 and BAP1). Additionally, survival analysis was conducted to assess difference between low and high groups of immune/stromal score.

\section{Identification of differentially expressed genes (DEGs) between low and high groups stratified by immune or stromal score}

DEGs between low and high groups were identified by performing data analysis according to limma package from $\mathrm{R}$ software(35). The following cut-off points were adopted to screen DEGs: $>1.5$ of fold change with adjusted $P$ value $<0.05$. Heatmaps, volcano plot and venn diagram were plotted by $R$ software using pheatmap package, ggplot2 and gplots.

\section{Functional enrichment analysis of DEGs}

DAVID (The Database for Annotation, Visualization and Integrated Discovery) is a classic database (https://david.ncifcrf.gov/) mainly used for gene functional enrichment analysis(36). DAVID provides a comprehensive annotation of biological function, and we used the database to conduct gene ontology (GO) functional annotation and Kyoto encyclopedia of genes and genomes (KEGG) pathway analysis. GO annotation included three parts: biological process (BP), molecular function (MF) and cell composition (CC). Visual results of the above analysis were presented by bubble plot conducted by using $\mathrm{R}$ software with ggplot2 package. $\mathrm{P}$ value $<0.05$ was considered as cut-off point of statistically significant. 


\section{Construction of protein-protein interaction (PPI) network and module analysis}

We used a biological database named STRING (Search Tool for the Retrieval of Interacting Genes) for PPI analysis. The role of the database (http://string.embl.de/) was to conduct a network of PPI for identified DEGs on the basis of the proven and predicted PPIs, and then analyze the potential interactions among proteins(37). After online analysis, TSV format files were downloaded for further analysis in Cytoscape software (version 3.5.1)(38). We used Cytoscape software to visualize PPI network and explore top 3 significant modules of network with 10 or more nodes.

\section{Survival analysis}

Analysis of overall outcome was performed by R software with survival package. Additionally, KaplanMeier plots were conducted by R software with survminer package. We calculated hazard ratio (HR) and log-rank $P$ value for presentation. $P$ value $<0.05$ was considered as cut-off point of statistically significant.

The composition differences of 22 immune cell subtypes between low and high immune scores

In order to quantify the abundance of 22 immune subtypes in cCRCC specimens, the CIBERSORT deconvolution algorithm was applied(39). Specifically, gene expression data was uploaded to the CIBERSORT web portal (http://cibersort.stanford.edu/) to calculate proportions of immune cells using the LM22 signature at 1,000 permutations. After acquisition of the infiltration data of each case, we planned to find the difference of abundance of immune infiltration cell between low and high groups of immune scores.

\section{Results}

\section{Immune scores were significantly correlated with clinicopathological parameters and OS}

Among all 537 cases downloaded from TCGA portal, 191 (35.6\%) cases were female, 346 (64.4\%) cases were male. The median age was 61y (ranged from 26 to $90 \mathrm{y}$ ). According to ESTIMATE algorithm mentioned before, the distributions of immune and stromal scores were ranged from -11.57.91 to 2030.4 and from 1158.94 to 3076.4 , respectively. As presented in Figure 1, cases with T stage $\geq 2$ were associated with higher immune score rather than stromal score (Figure 1A-B). Tumor stage was not statistically different in both immune and stromal scores (Figure 1C-D). For histological grade, immune scores increased with escalation of nuclear grade (Figure 1E), whereas no such trend was observed in stromal scores (Figure 1F). 
From data of TCGA we observed top 4 mutated genes: VHL, PBRM1, SETD2 and BAP1. Except for BAP1, immune scores were not correlated with mutations of VHL, PBRM1 and SETD2, while stromal scores were not statistically associated with all four genes (Figure 1G-N). We further analyzed the correlations of immune and stromal scores with OS, and Kaplan-Meier survival curves showed that cases with high immune scores were associated poorer OS than cases with low immune scores (Figure 10), whereas stromal scores were not correlated with os (Figure 1P).

Gene expression profiles were distinct between low and high groups stratified by immune and stromal scores

As shown in Figure 2A-C, the gene expression profiles presented by RNAseq data were significantly different between low and high groups in both immune and stromal scores. Based on immune scores, a total of 1433 genes were up-regulated and 1910 genes were down-regulated. For stromal scores, the numbers of up-regulated and down-regulated genes were 230 and 235, respectively. The numbers of upregulated and down-regulated genes in both immune and stromal scores were 722 and 77 , respectively (Figure 2D-E).

It is worth noting that DEGs identified from comparing low and high groups based on immune scores were relatively independent from DEGs from stromal scores. Based on the prognosis results of immune and stromal scores (Figure 10-P), stromal scores had no value in predicting prognosis. Therefore, we conducted all subsequent analysis based on DEGs identified from immune scores in the present study. Functional enrichment analysis of the 1433 up-regulated genes revealed that these genes were mainly involved in the biological process of immune and inflammatory response (Figure 2F), the cellular components of plasma membrane (Figure $2 \mathrm{G}$ ), the molecular function of carbohydrate binding (Figure $2 \mathrm{H}$ ) and the KEEG pathway of cytokine-cytokine receptor interaction (Figure 2I).

\section{Individual DEGs and OS}

We explored the relationships of the 1433 up-regulated genes to OS and a total of 890 DEGs were statistically significant (Supplementary Table S1). Among them, 855 genes were shown to predict poor OS and only 35 genes were shown to predict favorable OS. Kaplan-Meir survival curves of selected genes (Top 10 DEGs) were shown in Figure 3 and high expression of each gene was associated with poor OS.

\section{PPI network among prognostic DEGs}

We obtained the protein-protein interaction among DEGs by using the STRING tool. After downloading the data, we plotted PPI network through Cytoscape software for subsequent analysis. The whole network was composed of 733 nodes and 7001 edges, and a total of 7 modules were screened through MCODE. For further analysis, we selected the three most important modules (Figure 4). In the module 1, a total of 32 nodes and 495 edges form the network, and CXCL8, CXCR3/4/5 and CCL4/5 were the most important 
nodes (Figure 4A). In the module 2 (Figure 4B), 55 nodes involving 639 edges were formed in the network. The main nodes included IL6, IL10, CTLA4, FOXP3, and ITGAX. The module 3 was consisted of 47 nodes and 297 edges, including MMP9, STAT1, IL10RA and CD80 as the most important nodes (Figure 4C).

Functional enrichment analysis of DEGs with prognostic value and the role of top 10 hub genes in predicting OS

Functional enrichment analysis of the 890 DEGs revealed that these genes were mainly involved in immune and inflammatory response (Figure 5A), plasma membrane (Figure 5B), carbohydrate binding (Figure 5C) and cytokine-cytokine receptor interaction (Figure 5D), which were identical to the results of the 1433 up-regulated genes and PPI network analysis.

We further classified cases into two groups based on number of up-regulated genes in Top 10 DEGs (IL10RA, FCER1G, SASH3, TIGIT, RHOH, IL12RB1, AIF1, LPXN, LAPTM5 and SP140). Figure 5E showed the heatmap of these genes between low and high immune score groups. Kaplan-Meir survival curve was plotted to verify the prognostic value of the classification. Cases with number of up-regulated genes $\geq 5$ were associated poor OS ( $P=0.002$, Figure $5 F)$.

The compositions of CD8 T cells, CD4 memory resting T cells and mast resting cells were significant different between low and high immune scores

Among 22 subtypes of immune infiltration cells, up to $72.7 \%$ (16/22) of cells were statistically different between low and high immune scores (Figure 6). The mean differences of percentages of CD8 T cells (11.32\%), CD4 memory resting T cells $(-4.52 \%)$ and mast resting cells $(-3.55 \%)$ between low and high immune scores were the most significant, with CD8 T cells the greatest (increased from $7.64 \%$ to $18.96 \%$, $P<0.001)$. It is noteworthy that the trend of change in cell composition of CD8 T cells were opposite to CD4 memory resting T cells and mast resting cells (Figure 6).

\section{Discussion}

As the in-depth research on molecular mechanism of incidence and evolution of solid tumors including renal cell carcinoma, researchers used ICls to block co-inhibitory signals, prevent inactivation of $\mathrm{T}$ cells and then facilitate $T$ cells to kill tumor cells $(40,41)$. The unsatisfied treatment response keeps impelling researchers concentrate on developing and optimizing biomarkers. Current biomarkers focusing on immune status and prognostic models were not sufficient in screening out appropriate patients to receive ICls treatment. Thus, it is important to focus on the comprehensive immune status in TME. Based on this, we aimed to predict the prognosis of ccRCC by calculating immune and stromal scores in the aspect of comprehensive level, and explore potential immune related genes to predict prognosis of ccRCC.

In the present study, we found that immune score was a poor prognostic factor of OS. Since stromal score was not associated with prognosis, all subsequent analysis was based on immune score. After comparing gene expression profiles of cases with low and high scores, a total of 1433 genes related to 
immune response were identified. Functional enrichment analysis showed that most of these genes were involved in tumor microenvironment. Further survival analysis revealed that 890 genes were found to be associated with prognosis, and PPI network analysis also found that these genes were associated with immune or inflammatory responses, such as IL6, IL10, CTLA4, FOXP3, and ITGAX in module 1. Eventually, we identified top 10 prognosis-related DEGs to be integrated as one comprehensive parameter to predict prognosis of ccRCC. The comprehensive parameter was consisted of IL10RA, FCER1G, SASH3, TIGIT, RHOH, IL12RB1, AIF1, LPXN, LAPTM5 and SP140. Kaplan-Meier survival analysis suggested that patients with number of up-regulated genes $\geq 5$ have shorter OS compared to patients with number of upregulated genes $<5$.

The top 10 prognosis-related DEGs were found to be mainly correlated with immune and inflammatory process. IL10RA (Interleukin 10 Receptor Subunit Alpha) was mainly expressed on most hematopoietic cells and functioned as anti-inflammatory factor(42, 43). FCER1G (Fc Fragment Of IgE Receptor Ig) were associated with allergic reactions and played a role in tumor development and squamous carcinogenesis $(44,45)$. In addition, FCER1G was associated with progression and prognosis of cCRCC(46). Mutations or abnormal expressions of SASH3 (SAM And SH3 Domain Containing 3), RHOH (Ras Homolog Family Member H), LPXN (leupaxin) and LAPTM5 (lysosomal protein transmembrane 5) have been demonstrated to be associated with the development and progression multiple malignancies(47-56). TIGIT (T cell immunoglobulin and ITIM domain), IL12RB1(Interleukin 12 Receptor Subunit Beta 1), AIF1 (allograft inflammatory factor 1) and SP140 (SP140 nuclear body protein) mainly involved in the process of immune and inflammation. As a novel immune checkpoint receptor similar to PD-1, TIGIT is upregulated on CD8+ TILs and Tregs in multiple tumors, exhibiting therapeutic benefits in animal models $(57,58)$. IL12RB1 promotes delayed type hypersensitivity and autoimmunity(59). AIF1 is mainly associated with allograft rejection, autoimmune diseases and vasculopathy, etc.(60). SP140 was demonstrated to be associated with multiple sclerosis(61).

We also compared subtypes of immune cells between low and high immune scores to find potential factors attributed to the situation of high immune score. It seemed that increased compositions of CD8 T cells and decreased proportions of CD4 memory resting $T$ cells and mast resting cells were the most important in contributing to higher immune score. Therefore, the higher the immune score, the worse the prognosis may be mainly related to CD8 T cells, which is consistent with previous research evidence, suggesting that the reactions of immune cells were more pronounced as the progression of tumor grade/biological malignancy, possibly due to the increased antigenicity of tumor cells $(62,63)$.

Immunohistochemistry (IHC), flow cytometry (FCM) and next-gene sequencing (NGS) were the three major tools in assessment of immune status. Previous reports on the relationship between immune status and prognosis were mainly based on IHC and FCM, which focused on single or partial subtypes of immune cells(64-66). It has been found that immune scores (scored by densities of CD3+ and CD8+ by $\mathrm{IHC})$ can accurately estimate the risk of recurrence in colon cancer, and these results support the inclusion of immune scores as a new component of the TNM immune cancer classification(67). In terms of ccRCC, Kawashima et al classified patients' immune status into 3 groups using 5 markers (PD-1, 
TIM-3, ICOS, CD45RA, and CD25). The classification of the above 3 groups was significantly associated with tumor grade(68). Theoretically, the prediction of immunotherapy efficacy based on molecular markers of tumor and/or immune cells alone is not comprehensive and the clinical effect is mostly not satisfactory. ICls are different from antiangiogenic drugs in that they act directly on tumor microenvironment rather than tumor cells. Therefore, a growing number of researchers are focusing on the predictive value of comprehensive evaluation. In the field of ccRCC, efforts have been made in characterizing immune status in a more comprehensive way. Xu et al has developed novel signatures of immune infiltration based on calculating immune and stromal scores(69). In the present study, we identified top 10 immune microenvironment-related genes and these genes were integrated into one parameter to represent immune status. Survival analysis demonstrated that this integrated parameter can distinguish patients with good or poor prognosis. However, further researches are warrant to explore the potential of predictive value of these genes in the immunotherapy of ccRCC.

\section{Abbreviations}

clear cell renal cell carcinoma (ccRCC)

differentially expressed genes (DEGs)

immune checkpoint inhibitors (ICls)

tumor mutation burden (TMB)

tumor neoantigen burden (TNB)

cytolytic activity (CYT)

tumor microenvironment (TME)

regulatory $T$ cells (Tregs)

tumor associated macrophages (TAM)

the cancer genome atlas (TCGA)

Immunohistochemistry (IHC)

flow cytometry (FCM)

next-gene sequencing (NGS)

gene ontology (GO)

Kyoto encyclopedia of genes and genomes (KEGG) 
biological process (BP)

molecular function (MF)

cell composition (CC).

\section{Declarations}

Ethics approval and consent to participate: Not applicable

Consent for publication: Not applicable

Availability of data and materials:

The datasets analyzed during the current study are available in the TCGA database (https://tcgadata.nci.nih.gov/tcga/).

The datasets analyzed during the current study are available in DAVID database (The Database for Annotation, Visualization and Integrated Discovery) (https://david.ncifcrf.gov/).

The datasets analyzed during the current study are available in STRING (Search Tool for the Retrieval of Interacting Genes) database (http://string.embl.de/).

Competing interests: The authors declare that they have no competing interests.

Funding: This work was supported by Natural Science Foundation of China (NSFC 81974398, 81902577 and 81672547 ) and 1.3.5 project for disciplines of excellence, West China Hospital, Sichuan University (No.0040205301E21).

Authors' contributions: JW and $\mathrm{HZ}$ proposed the concept of this study; XMZ and XXY analyzed the database and were major contributors in writing the original manuscript. XDZ, HRZ, JGZ and JRC were mainly in charge of data search, selection, and study quality assessment. ZHL, SZ, GXS, NC and PFS were major contributors in writing review. All authors read and approved the final manuscript.

Acknowledgements: Not applicable

\section{References}

1.Rini BI, Campbell SC, Escudier B. Renal cell carcinoma. Lancet. 2009;373(9669):1119-32.

2.Thompson $\mathrm{RH}$, Dong H, Lohse $\mathrm{CM}$, Leibovich BC, Blute ML, Cheville JC, et al. PD-1 is expressed by tumor-infiltrating immune cells and is associated with poor outcome for patients with renal cell carcinoma. Clin Cancer Res. 2007;13(6):1757-61. 
3.Janiszewska AD, Poletajew S, Wasiutynski A. Spontaneous regression of renal cell carcinoma. Contemporary oncology (Poznan, Poland). 2013;17(2):123-7.

4.Atkins MB, Regan M, McDermott D. Update on the role of interleukin 2 and other cytokines in the treatment of patients with stage IV renal carcinoma. Clin Cancer Res. 2004;10(18 Pt 2):6342s-6s.

5.Inman BA, Harrison MR, George DJ. Novel immunotherapeutic strategies in development for renal cell carcinoma. Eur Urol. 2013;63(5):881-9.

6. Herbst RS, Soria JC, Kowanetz M, Fine GD, Hamid O, Gordon MS, et al. Predictive correlates of response to the anti-PD-L1 antibody MPDL3280A in cancer patients. Nature. 2014;515(7528):563-7.

7.Motzer RJ, Tannir NM, McDermott DF, Aren Frontera O, Melichar B, Choueiri TK, et al. Nivolumab plus Ipilimumab versus Sunitinib in Advanced Renal-Cell Carcinoma. N Engl J Med. 2018;378(14):1277-90.

8.Motzer RJ, Escudier B, McDermott DF, George S, Hammers HJ, Srinivas S, et al. Nivolumab versus Everolimus in Advanced Renal-Cell Carcinoma. N Engl J Med. 2015;373(19):1803-13.

9.Motzer RJ, Penkov K, Haanen J, Rini B, Albiges L, Campbell MT, et al. Avelumab plus Axitinib versus Sunitinib for Advanced Renal-Cell Carcinoma. N Engl J Med. 2019.

10.Rini BI, Plimack ER, Stus V, Gafanov R, Hawkins R, Nosov D, et al. Pembrolizumab plus Axitinib versus Sunitinib for Advanced Renal-Cell Carcinoma. N Engl J Med. 2019.

11.David F M, Lee. J-L, Szczylik. C, Donskov. F, Malik. J, Alekseev. BY, et al. Pembrolizumab monotherapy as first-line therapy in advanced clear cell renal cell carcinoma (accRCC): Results from cohort A of KEYNOTE-427. J Clin Oncol. 2018;36(suppl):abstr 4500.

12.Robert J M, Powles. T, Michael B A, Escudier. B, David F M, Suarez. C, et al. IMmotion151: A Randomized Phase III Study of Atezolizumab Plus Bevacizumab vs Sunitinib in Untreated Metastatic Renal Cell Carcinoma (mRCC). J Clin Oncol. 2018;36(suppl):abstr 578.

13.Motzer RJ, Rini BI, McDermott DF, Redman BG, Kuzel TM, Harrison MR, et al. Nivolumab for Metastatic Renal Cell Carcinoma: Results of a Randomized Phase II Trial. J Clin Oncol. 2015;33(13):1430-7.

14.Carbognin L, Pilotto S, Milella M, Vaccaro V, Brunelli M, Calio A, et al. Differential Activity of Nivolumab, Pembrolizumab and MPDL3280A according to the Tumor Expression of Programmed Death-Ligand-1 (PD-L1): Sensitivity Analysis of Trials in Melanoma, Lung and Genitourinary Cancers. PLoS One. 2015;10(6):e0130142.

15.Correa AF, Jegede O, Haas NB, Flaherty KT, Pins MR, Messing EM, et al. Predicting Renal Cancer Recurrence: Defining Limitations of Existing Prognostic Models With Prospective Trial-Based Validation. J Clin Oncol. 2019;37(23):2062-71. 
16.Lawrence MS, Stojanov P, Polak P, Kryukov GV, Cibulskis K, Sivachenko A, et al. Mutational heterogeneity in cancer and the search for new cancer-associated genes. Nature. 2013;499(7457):214-8.

17.Turajlic S, Litchfield K, Xu H, Rosenthal R, McGranahan N, Reading JL, et al. Insertion-and-deletionderived tumour-specific neoantigens and the immunogenic phenotype: a pan-cancer analysis. Lancet Oncol. 2017;18(8):1009-21.

18.McDermott DF, Huseni MA, Atkins MB, Motzer RJ, Rini BI, Escudier B, et al. Clinical activity and molecular correlates of response to atezolizumab alone or in combination with bevacizumab versus sunitinib in renal cell carcinoma. Nat Med. 2018;24(6):749-57.

19.Rooney MS, Shukla SA, Wu CJ, Getz G, Hacohen N. Molecular and genetic properties of tumors associated with local immune cytolytic activity. Cell. 2015;160(1-2):48-61.

20.Pitt JM, Marabelle A, Eggermont A, Soria JC, Kroemer G, Zitvogel L. Targeting the tumor microenvironment: removing obstruction to anticancer immune responses and immunotherapy. Ann Oncol. 2016;27(8):1482-92.

21. Hinshaw DC, Shevde LA. The Tumor Microenvironment Innately Modulates Cancer Progression. Cancer Res. 2019.

22. Hanahan D, Coussens LM. Accessories to the crime: functions of cells recruited to the tumor microenvironment. Cancer Cell. 2012;21(3):309-22.

23.Nishikawa H, Sakaguchi S. Regulatory T cells in cancer immunotherapy. Curr Opin Immunol. 2014;27:1-7.

24.Noy R, Pollard JW. Tumor-associated macrophages: from mechanisms to therapy. Immunity. 2014;41(1):49-61.

25.Gajewski TF, Schreiber H, Fu YX. Innate and adaptive immune cells in the tumor microenvironment. Nat Immunol. 2013;14(10):1014-22.

26.Galon J, Costes A, Sanchez-Cabo F, Kirilovsky A, Mlecnik B, Lagorce-Pages C, et al. Type, density, and location of immune cells within human colorectal tumors predict clinical outcome. Science. 2006;313(5795):1960-4.

27.Pages F, Kirilovsky A, Mlecnik B, Asslaber M, Tosolini M, Bindea G, et al. In situ cytotoxic and memory T cells predict outcome in patients with early-stage colorectal cancer. J Clin Oncol. 2009;27(35):5944-51.

28.Choueiri TK, Fishman MN, Escudier B, McDermott DF, Drake CG, Kluger H, et al. Immunomodulatory Activity of Nivolumab in Metastatic Renal Cell Carcinoma. Clin Cancer Res. 2016;22(22):5461-71. 
29.Senbabaoglu Y, Gejman RS, Winer AG, Liu M, Van Allen EM, de Velasco G, et al. Tumor immune microenvironment characterization in clear cell renal cell carcinoma identifies prognostic and immunotherapeutically relevant messenger RNA signatures. Genome Biol. 2016;17(1):231.

30.Chen YP, Zhang Y, Lv JW, Li YQ, Wang YQ, He QM, et al. Genomic Analysis of Tumor Microenvironment Immune Types across 14 Solid Cancer Types: Immunotherapeutic Implications. Theranostics. 2017;7(14):3585-94.

31.Carter SL, Cibulskis K, Helman E, McKenna A, Shen H, Zack T, et al. Absolute quantification of somatic DNA alterations in human cancer. Nat Biotechnol. 2012;30(5):413-21.

32. Yoshihara K, Shahmoradgoli M, Martinez E, Vegesna R, Kim H, Torres-Garcia W, et al. Inferring tumour purity and stromal and immune cell admixture from expression data. Nature communications. 2013;4:2612.

33.Song E, Song W, Ren M, Xing L, Ni W, Li Y, et al. Identification of potential crucial genes associated with carcinogenesis of clear cell renal cell carcinoma. 2018;119(7):5163-74.

34.Linehan WM, Ricketts CJ. The Cancer Genome Atlas of renal cell carcinoma: findings and clinical implications. Nature reviews Urology. 2019.

35.Ritchie ME, Phipson B, Wu D, Hu Y, Law CW, Shi W, et al. limma powers differential expression analyses for RNA-sequencing and microarray studies. Nucleic Acids Res. 2015;43(7):e47.

36. Huang da W, Sherman BT, Lempicki RA. Systematic and integrative analysis of large gene lists using DAVID bioinformatics resources. Nat Protoc. 2009;4(1):44-57.

37.Szklarczyk D, Franceschini A, Wyder S, Forslund K, Heller D, Huerta-Cepas J, et al. STRING v10: proteinprotein interaction networks, integrated over the tree of life. Nucleic Acids Res. 2015;43(Database issue):D447-52.

38.Shannon P, Markiel A, Ozier O, Baliga NS, Wang JT, Ramage D, et al. Cytoscape: a software environment for integrated models of biomolecular interaction networks. Genome Res. 2003;13(11):2498-504.

39.Newman AM, Liu CL, Green MR. Robust enumeration of cell subsets from tissue expression profiles. 2015;12(5):453-7.

40.Sharma P, Allison JP. The future of immune checkpoint therapy. Science. 2015;348(6230):56-61.

41.Atkins MB, Clark JI, Quinn DI. Immune checkpoint inhibitors in advanced renal cell carcinoma: experience to date and future directions. Ann Oncol. 2017. 
42.Hutchins AP, Diez D, Miranda-Saavedra D. The IL-10/STAT3-mediated anti-inflammatory response: recent developments and future challenges. Briefings in functional genomics. 2013;12(6):489-98.

43.Shouval DS, Ouahed J, Biswas A, Goettel JA, Horwitz BH, Klein C, et al. Interleukin 10 receptor signaling: master regulator of intestinal mucosal homeostasis in mice and humans. Adv Immunol. 2014;122:177-210.

44.Tsuji M, Ezumi Y, Arai M, Takayama H. A novel association of Fc receptor gamma-chain with glycoprotein $\mathrm{VI}$ and their co-expression as a collagen receptor in human platelets. J Biol Chem. 1997;272(38):23528-31.

45.Andreu P, Johansson M, Affara NI, Pucci F, Tan T, Junankar S, et al. FcRgamma activation regulates inflammation-associated squamous carcinogenesis. Cancer Cell. 2010;17(2):121-34.

46.Chen L, Yuan L, Wang Y, Wang G, Zhu Y, Cao R, et al. Co-expression network analysis identified FCER1G in association with progression and prognosis in human clear cell renal cell carcinoma. Int $\mathrm{J}$ Biol Sci. 2017;13(11):1361-72.

47.Li L, Peng M, Xue W, Fan Z, Wang T, Lian J, et al. Integrated analysis of dysregulated long non-coding RNAs/microRNAs/mRNAs in metastasis of lung adenocarcinoma. J Transl Med. 2018;16(1):372.

48.Schieffer KM, Choi CS, Emrich S, Harris L, Deiling S, Karamchandani DM, et al. RNA-seq implicates deregulation of the immune system in the pathogenesis of diverticulitis. Am J Physiol Gastrointest Liver Physiol. 2017;313(3):G277-G84.

49.Sanati N, lancu OD, Wu G, Jacobs JE, McWeeney SK. Network-Based Predictors of Progression in Head and Neck Squamous Cell Carcinoma. Frontiers in genetics. 2018;9:183.

50.Erdem-Eraslan L, Heijsman D, de Wit M, Kremer A, Sacchetti A, van der Spek PJ, et al. Tumor-specific mutations in low-frequency genes affect their functional properties. J Neurooncol. 2015;122(3):461-70.

51.Troeger A, Williams DA. Hematopoietic-specific Rho GTPases Rac2 and RhoH and human blood disorders. Exp Cell Res. 2013;319(15):2375-83.

52.Voena C, Chiarle R. RHO Family GTPases in the Biology of Lymphoma. Cells. 2019;8(7).

53. Woldu SL, Hutchinson RC, Krabbe LM, Sanli O, Margulis V. The Rho GTPase signalling pathway in urothelial carcinoma. Nature reviews Urology. 2018;15(2):83-91.

54.Chen L, Wang G, Luo Y, Wang Y, Xie C, Jiang W, et al. Downregulation of LAPTM5 suppresses cell proliferation and viability inducing cell cycle arrest at G0/G1 phase of bladder cancer cells. Int J Oncol. 2017;50(1):263-71. 
55.Dierks S, von Hardenberg S, Schmidt T, Bremmer F, Burfeind P, Kaulfuss S. Leupaxin stimulates adhesion and migration of prostate cancer cells through modulation of the phosphorylation status of the actin-binding protein caldesmon. Oncotarget. 2015;6(15):13591-606.

56.Hou T, Zhou L, Wang L, Kazobinka G, Chen Y, Zhang X, et al. Leupaxin Promotes Bladder Cancer Proliferation, Metastasis, and Angiogenesis Through the PI3K/AKT Pathway. Cell Physiol Biochem. 2018;47(6):2250-60.

57.Zhang X, Zhang H, Chen L, Feng Z, Gao L, Li Q. TIGIT expression is upregulated in T cells and causes $T$ cell dysfunction independent of PD-1 and Tim-3 in adult B lineage acute lymphoblastic leukemia. Cell Immunol. 2019;344:103958.

58.Liu XG, Hou M, Liu Y. TIGIT, A Novel Therapeutic Target for Tumor Immunotherapy. Immunol Invest. 2017;46(2):172-82.

59.Robinson RT. IL12Rbeta1: the cytokine receptor that we used to know. Cytokine. 2015;71(2):348-59.

60.Zhao YY, Yan DJ, Chen ZW. Role of AlF-1 in the regulation of inflammatory activation and diverse disease processes. Cell Immunol. 2013;284(1-2):75-83.

61.Karaky M, Fedetz M, Potenciano V, Andres-Leon E, Codina AE, Barrionuevo C, et al. SP140 regulates the expression of immune-related genes associated with multiple sclerosis and other autoimmune diseases by NF-kappaB inhibition. Hum Mol Genet. 2018;27(23):4012-23.

62.Nakano O, Sato M, Naito Y, Suzuki K, Orikasa S, Aizawa M, et al. Proliferative activity of intratumoral CD8(+) T-lymphocytes as a prognostic factor in human renal cell carcinoma: clinicopathologic demonstration of antitumor immunity. Cancer Res. 2001;61(13):5132-6.

63.Mella M, Kauppila JH, Karihtala P, Lehenkari P, Jukkola-Vuorinen A, Soini Y, et al. Tumor infiltrating CD8(+) T lymphocyte count is independent of tumor TLR9 status in treatment naive triple negative breast cancer and renal cell carcinoma. Oncoimmunology. 2015;4(6):e1002726.

64.Bromwich EJ, McArdle PA, Canna K, McMillan DC, McNicol AM, Brown M, et al. The relationship between T-lymphocyte infiltration, stage, tumour grade and survival in patients undergoing curative surgery for renal cell cancer. Br J Cancer. 2003;89(10):1906-8.

65.Hotta K, Sho M, Fujimoto K, Shimada K, Yamato I, Anai S, et al. Prognostic significance of CD45RO+ memory T cells in renal cell carcinoma. Br J Cancer. 2011;105(8):1191-6.

66.Chevrier S, Levine JH, Zanotelli VRT, Silina K, Schulz D, Bacac M, et al. An Immune Atlas of Clear Cell Renal Cell Carcinoma. Cell. 2017;169(4):736-49 e18.

67.Pages F, Mlecnik B, Marliot F, Bindea G, Ou FS, Bifulco C, et al. International validation of the consensus Immunoscore for the classification of colon cancer: a prognostic and accuracy study. Lancet. 
2018;391(10135):2128-39.

68.Kawakami F, Sircar K, Rodriguez-Canales J, Fellman BM, Urbauer DL, Tamboli P, et al. Programmed cell death ligand 1 and tumor-infiltrating lymphocyte status in patients with renal cell carcinoma and sarcomatoid dedifferentiation. 2017;123(24):4823-31.

69.Xu WH, Xu Y, Wang J, Wan FN, Wang HK, Cao DL, et al. Prognostic value and immune infiltration of novel signatures in clear cell renal cell carcinoma microenvironment. Aging (Albany NY). 2019;11.

\section{Figures}



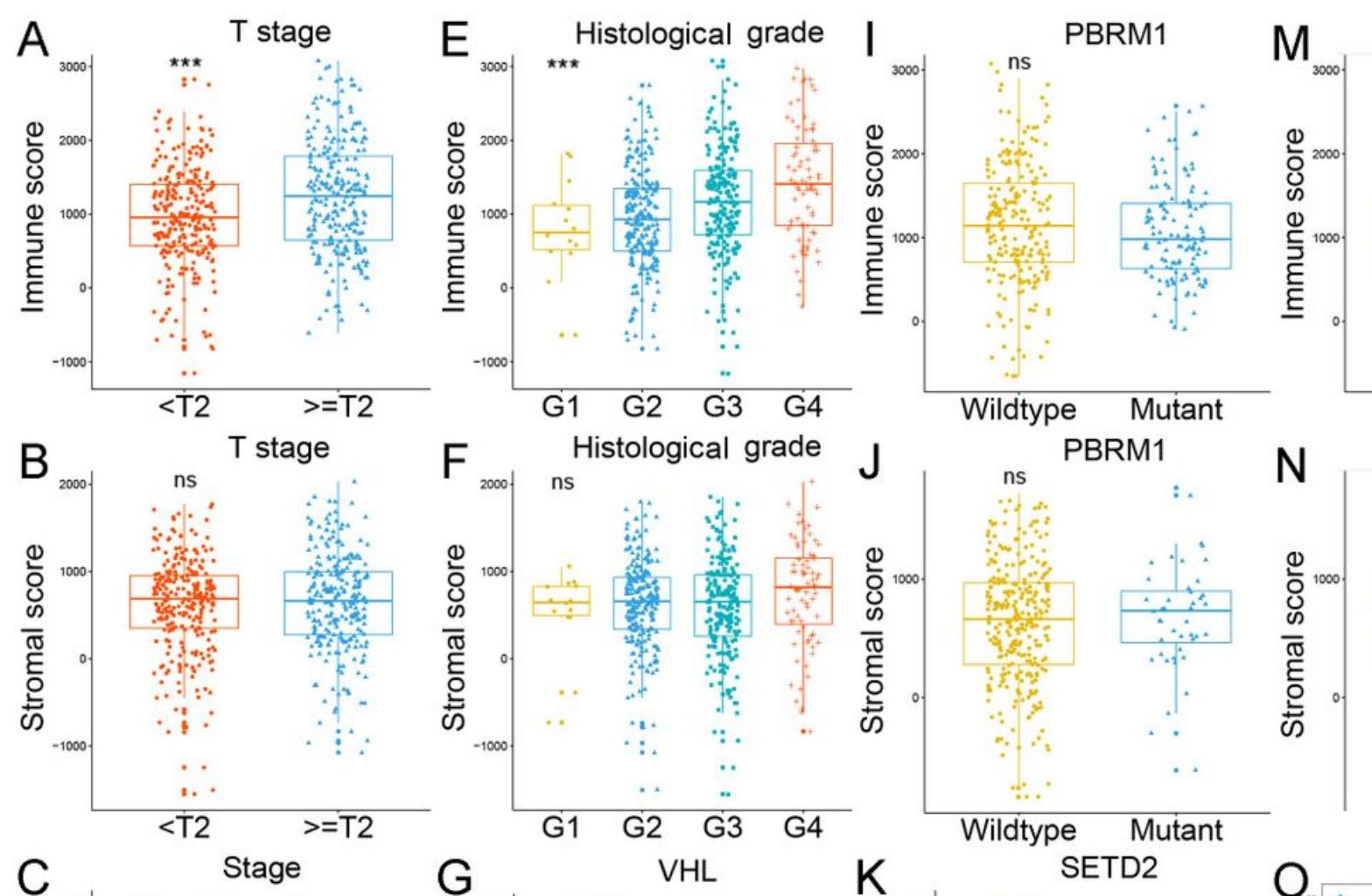

BAP1
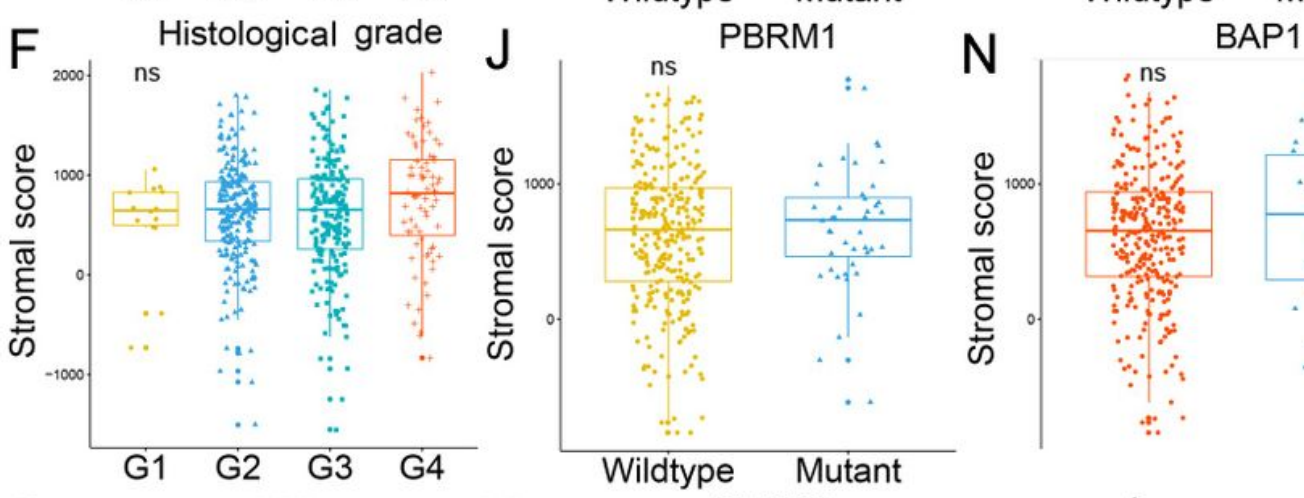

Wildtype Mutant
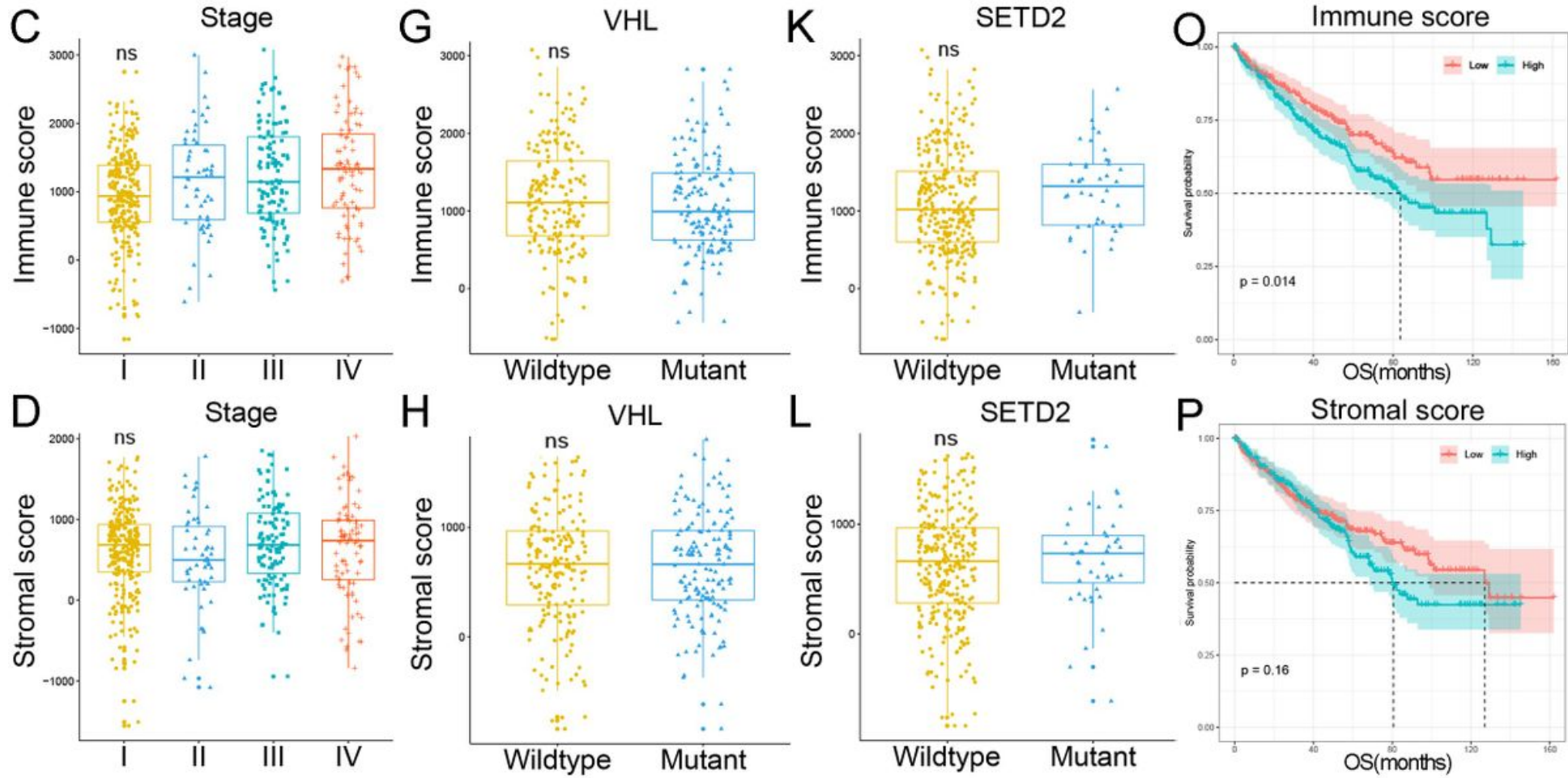

Figure 1

Immune scores were significantly correlated with clinicopathological parameters and OS A and B represented the associations of $\mathrm{T}$ stage with immune and stromal scores. $\mathrm{C}$ and $\mathrm{D}$ represented the associations tumor stage with immune and stromal scores. $\mathrm{E}$ and $\mathrm{F}$ indicated the associations of histological grade with immune and stromal scores. From $\mathrm{G}$ to $\mathrm{N}$, correlations of top 4 mutated genes of TCGA with immune and stromal scores were presented. We further analyzed the correlations of immune 
and stromal scores with OS. $\mathrm{O}$ and $\mathrm{P}$ showed Kaplan-Meier survival curves of immune and stromal scores for OS.
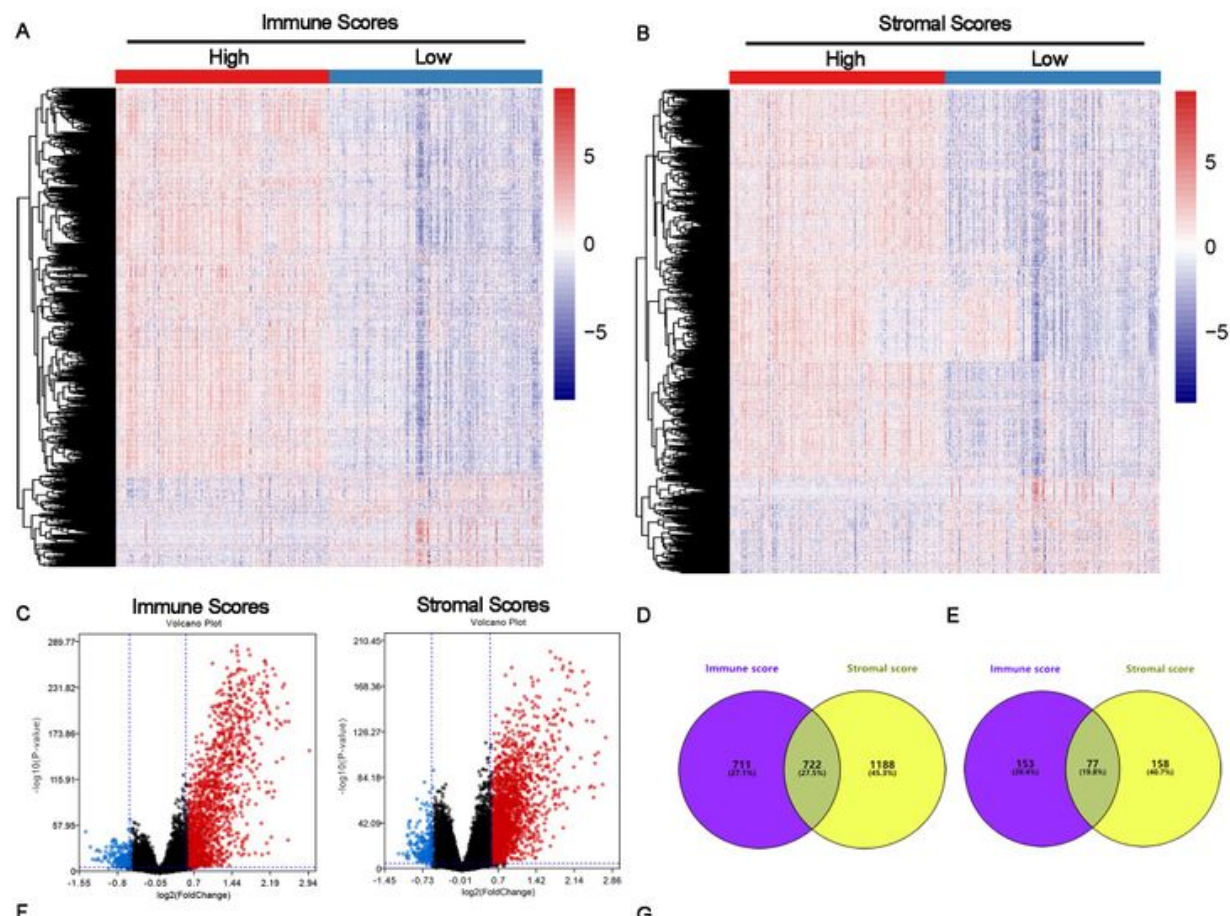

D
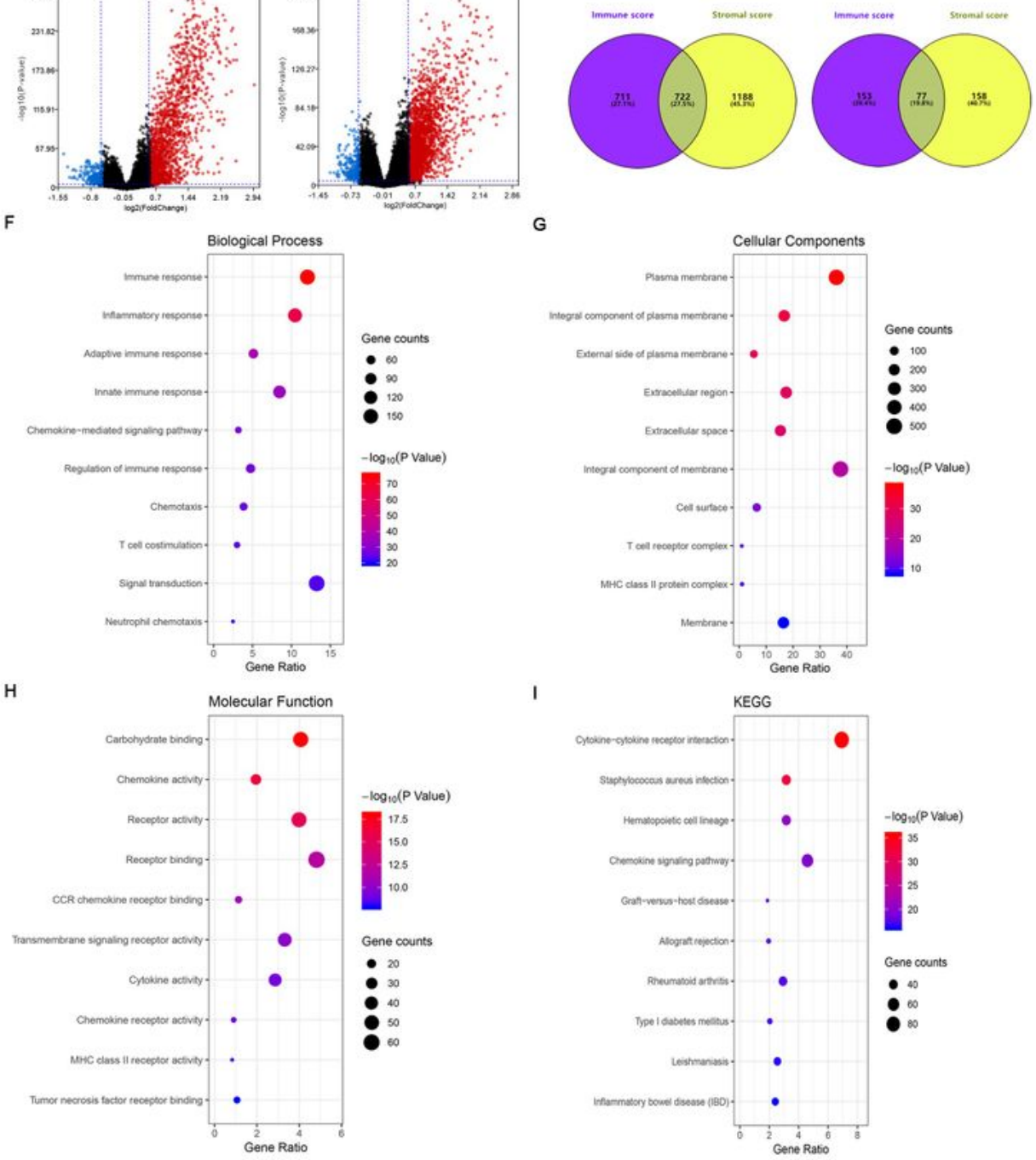

Figure 2

Gene expression profiles were distinct between low and high groups stratified by immune and stromal scores A-C showed that the gene expression profiles were significantly different between low and high groups in both immune and stromal scores. D-E represented numbers of genes up-regulated or down- 
regulated genes in both immune and stromal scores. F-I indicated functional enrichment analysis of the 1433 up-regulated genes.
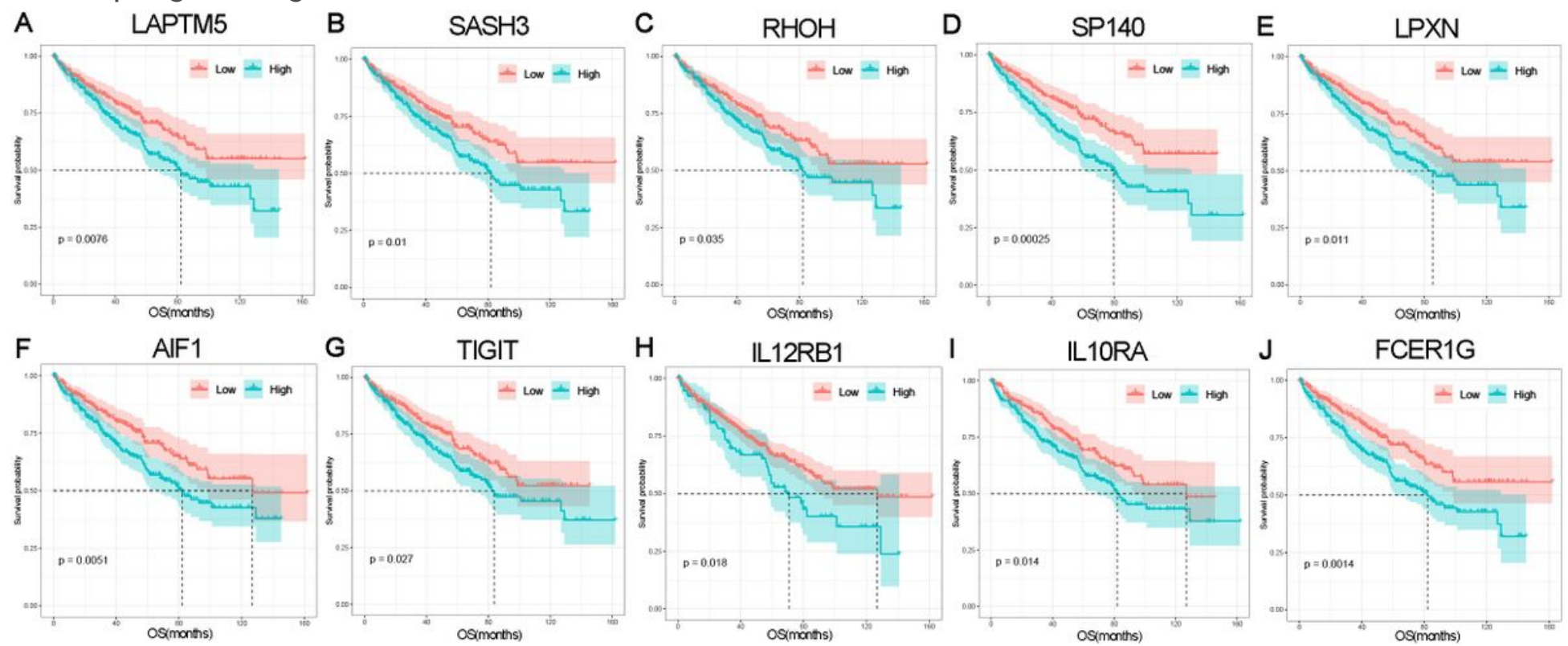

\section{Figure 3}

Associations of top 10 individual DEGs with OS

A

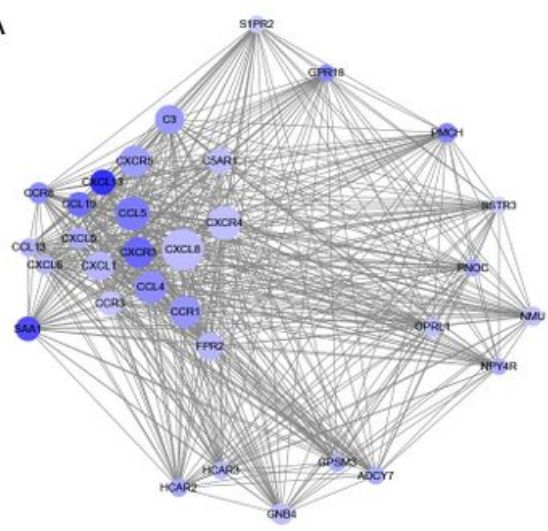

B

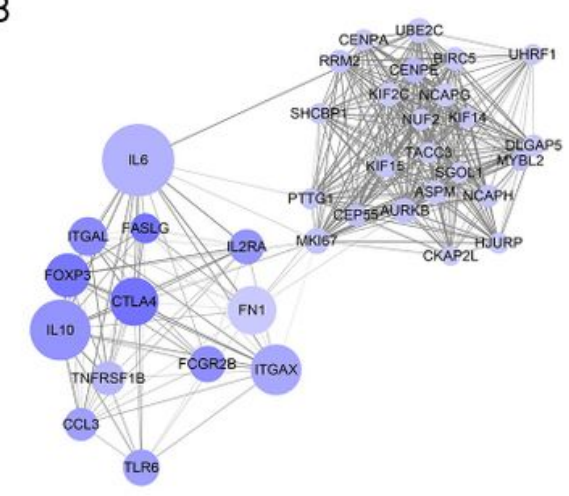

TuR
C



Figure 4

PPI network among prognostic DEGs A represented module 1, consisted of 32 nodes and 495 edges. $B$ represented module 2 , formed by 55 nodes and 639 edges. $C$ represented module 3 , involving 47 nodes and 297 edges. 

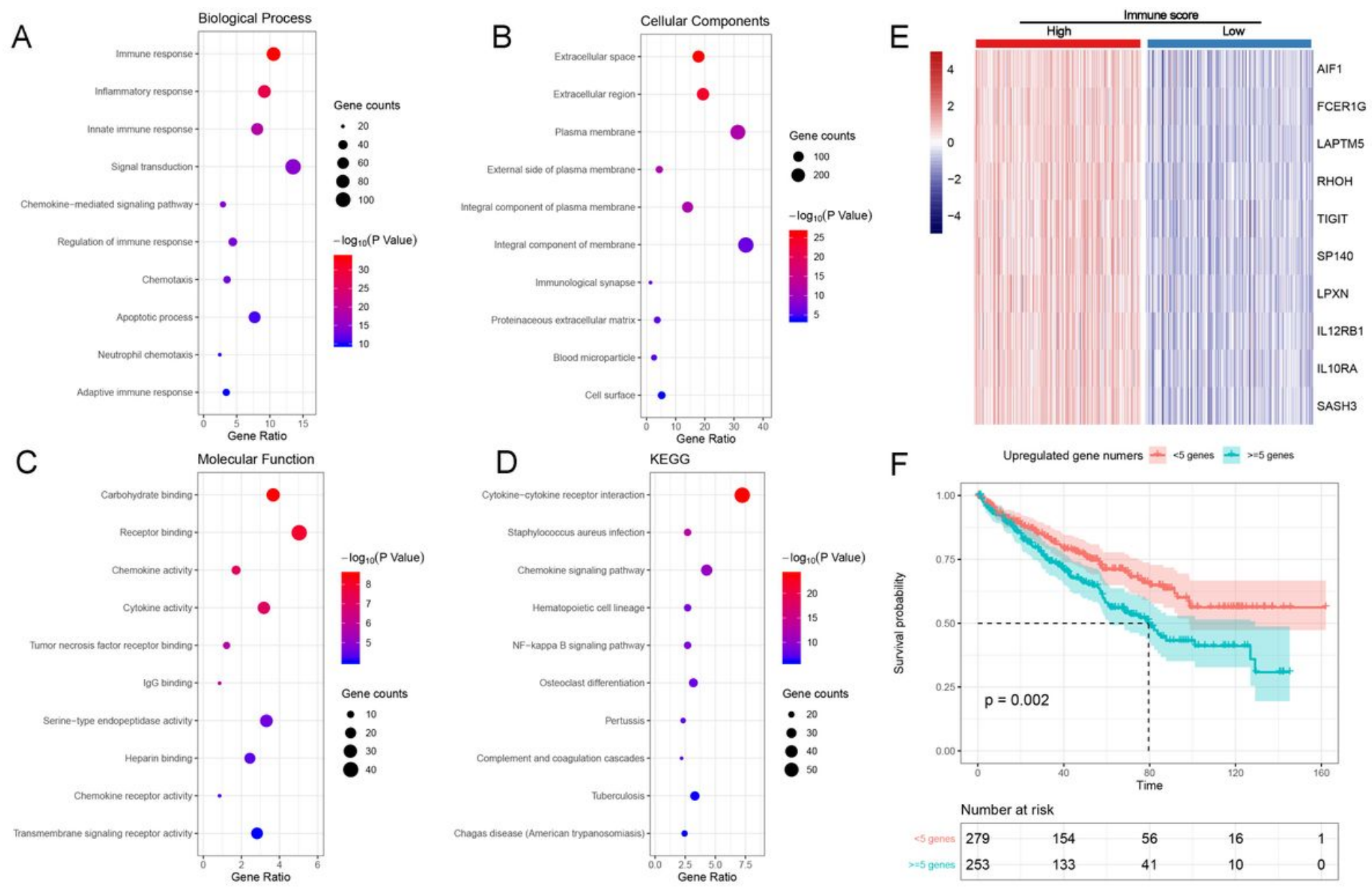

\section{Figure 5}

Functional enrichment analysis of DEGs with prognostic value and the role of top 10 hub genes in predicting OS A-D represented functional enrichment analysis. E represented the heatmap of these genes between low and high immune score groups and $\mathrm{F}$ represented Kaplan-Meir survival curve of OS in cases classified by number of up-regulated genes $\geq 5$ or not. 
$\bullet$ High $\bullet$ Low

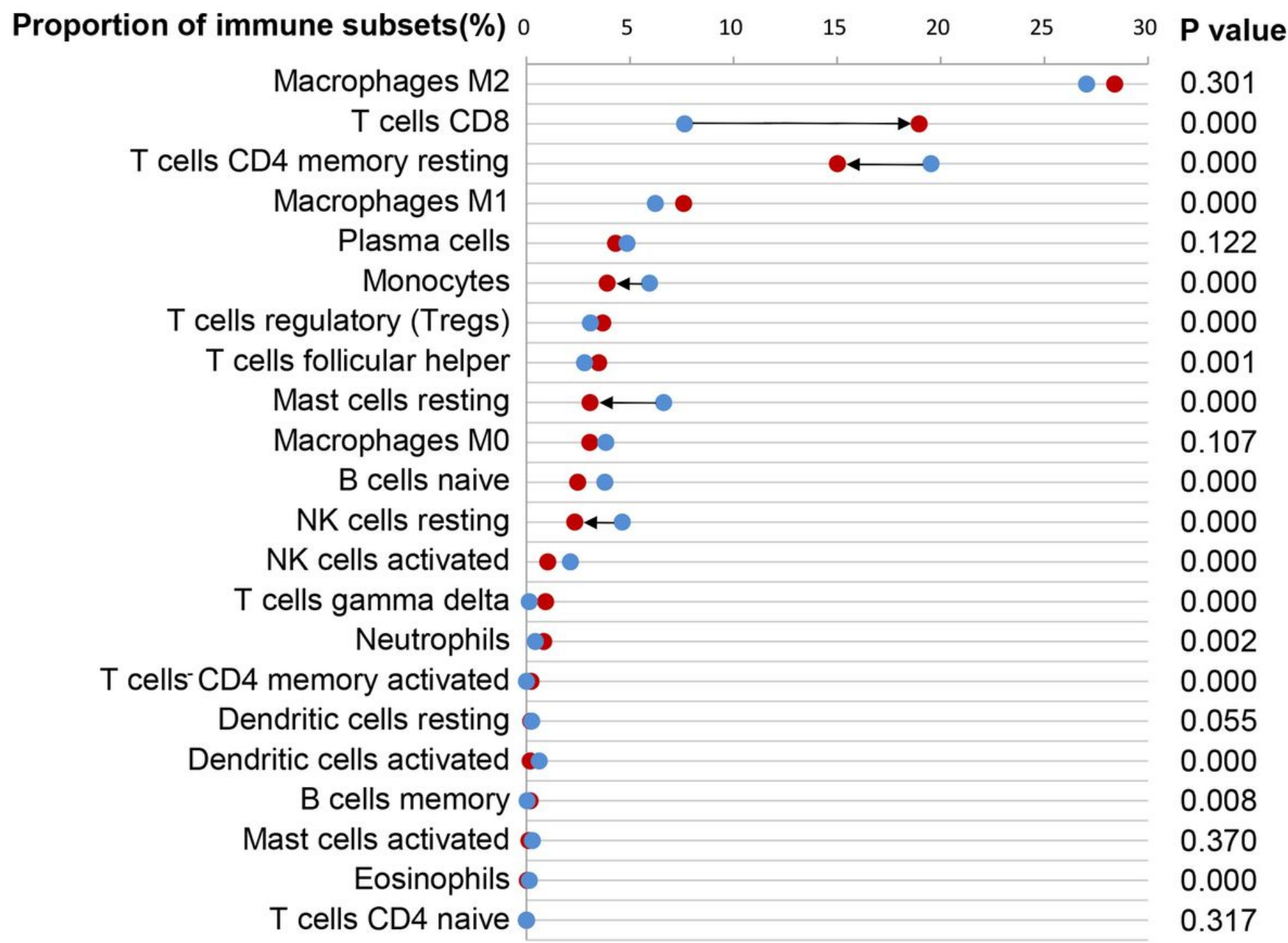

Figure 6

The compositions of CD8 T cells, CD4 memory resting T cells and mast resting cells were significant different between low and high immune scores

\section{Supplementary Files}

This is a list of supplementary files associated with this preprint. Click to download.

- SupplementaryTableS1.docx 